\title{
Modeling the Dynamics of Granular Matter
}

\author{
Rinaldo M. Colombo \\ Brescia University
}

\author{
Graziano Guerra \\ Milano-Bicocca University
}

\begin{abstract}
Francesca Monti
\end{abstract}
Milano-Bicocca University

November 5, 2009

\begin{abstract}
This work presents a new model for the movement of granular matter. It is a synthesis of the Hadeler-Kuttler and of the Savage-Hutter models. The result is a $3 \times 3$ system of balance laws able to describe the deposition-erosion dynamics, as in the former model, while being compliant with energy dissipation, as in the latter one. First, the basic analytical properties of the new model are described. Then, several numerical integrations allow to compare the different models. The present model appears to provide better descriptions of granular matter behaviour, in particular as soon as the bed's slope changes sign and deposition-erosion phenomena are relevant.
\end{abstract}

2000 Mathematics Subject Classification: 35L65, 76T25.

Keywords and phrases: Granular Flows, Hyperbolic models.

\section{Introduction}

Consider a slope with profile $u=u(t, x)$, where $x \in \mathbb{R}^{2}$ and $t \geq 0$. On this bed, some kind of material with thickness $h=h(t, x)$ is free to slide, subject to gravity. The sliding matter may well erode or deposit, thus modifying the slope as well as its distribution over it. This explains why both functions $u$ and $h$ are also time dependent. We describe the complex dynamics that arises through the following equations:

$$
\left\{\begin{array}{l}
\partial_{t} h+\nabla \cdot(h v)=-\gamma(\alpha-\|\nabla u\|) h+H \\
\partial_{t} v+\nabla \cdot\left(\frac{1}{2} v \otimes v+g h I\right)=-g \nabla u+\nu(v, \nabla u)-\gamma \llbracket \alpha-\|\nabla u\| \rrbracket{ }_{-} v+V \\
\partial_{t} u=\gamma(\alpha-\|\nabla u\|) h
\end{array}\right.
$$

Here, $v$ defines the velocity vector field at which matter slides over the bed. $g$ is gravity and $\alpha$ is the critical angle: at slopes higher than $\alpha$, the sliding 
matter erodes the bed while falling, whereas at lower slopes it deposits over the bed. The constant $\gamma$ is the speed at which erosion-deposition takes place. More precisely, in the first equation, the term $-\gamma h(\alpha-\|\nabla u\|)$ corresponds to the quantity of matter that deposits, when $\|\nabla u\|<\alpha$, or that is eroded, when $\|\nabla u\|>\alpha$. Conservation of mass requires that the same term appears, with the opposite sign, in the third equation. The right hand side of the second equation contains a first term $-g \nabla u$, describing the component of gravity parallel to the slope. The vector $\nu(v, \nabla u)$ describes the friction between the sliding matter and the slope. Therefore, we require the following physically obvious conditions:

$$
v \cdot \nu(v, \nabla u) \leq 0 \quad \text { and } \quad \nu(0, \nabla u)=0
$$

for all $v$ and $u$. The term $-\llbracket \gamma(\alpha-\|\nabla u\|) \rrbracket_{-} v$ in the second equation is due to the eroded material that starts moving and affects the speed of the sliding matter. Finally, $H$ and $V$ are functions of $(t, x)$, presumably known, modeling sources such as material falling or being poured over the bed.

A priori, the first two equations in (1.1) can be justified through the balance of mass and of linear momentum, see [8], the third equation by the erosion-deposition dynamics. Part of these terms, in fact, are found also in the Hadeler-Kuttler model introduced in [11, formula (5)]:

$$
\left\{\begin{array}{l}
\partial_{t} h-\nabla \cdot(\beta h \nabla u)=-\gamma(\alpha-\|\nabla u\|) h+H \\
\partial_{t} u=\gamma(\alpha-\|\nabla u\|) h
\end{array}\right.
$$

where we used the same notations as above. $\beta$ is the rate that connects the velocity of the rolling layer to the gradient of $u$, see [12]. This model has been widely considered in the literature, both from the point of view of stationary asymptotic solutions and from that of evolution problems, see for instance $[1,2,3,4,6,9,18]$. Some of the above studies are purely analytical, others are of a more numerical nature.

On the other hand, the convective part in the first two equations of (1.1) reminds of that of the Savage-Hutter model, see [15, 19], which, for smooth solutions, can be rewritten as

$$
(\mathbf{S H}) \quad\left\{\begin{array}{l}
\partial_{t} h+\nabla \cdot(h v)=0 \\
\partial_{t} v+\nabla \cdot\left(\frac{1}{2} v \otimes v+h G\right)=s .
\end{array}\right.
$$

Here, the vector $s$ and the $2 \times 2$ diagonal matrix $G$ are functions of the unknowns and of the space variables, see [19, formulæ (1)-(10)], whose role is to accurately describe the given fixed geometry of the slope and the effect of gravity. Remark that ( $\mathbf{S H})$ is essentially equivalent to the shallow water equations with a bed having a given fixed geometry and a drift term in the 
moment equation, see [8]. Several papers consider the model (SH) from various points of view, see for instance $[10,14,16,17]$.

Below, we study (1.1) and compare it with (HK) and (SH). To this aim, we first scale out the various constant parameters in (1.1) and (HK), obtaining

$$
\left\{\begin{array}{l}
\partial_{t} h+\nabla \cdot(h v)=-(1-\|\nabla u\|) h+H \\
\partial_{t} v+\nabla \cdot\left(\frac{1}{2} v \otimes v+h I\right)=-\nabla u+\nu(v, \nabla u)-\llbracket 1-\|\nabla u\| \rrbracket_{-} v+V \\
\partial_{t} u=(1-\|\nabla u\|) h
\end{array}\right.
$$

in the case of model (1.1), see Lemma 5.2. In the case of (HK), Lemma 5.1 yields the rescaling

$$
\left\{\begin{array}{l}
\partial_{t} h-\nabla \cdot(h \nabla u)=-(1-\|\nabla u\|) h+H \\
\partial_{t} u=(1-\|\nabla u\|) h .
\end{array}\right.
$$

A first key difference between (1.1) and (HK) is the energy balance. Indeed, smooth solutions to (1.1) dissipate the energy

$$
E=\int_{\mathbb{R}^{2}}\left(\frac{1}{2} h\|v\|^{2}+\frac{1}{2}(h+u)^{2}\right) \mathrm{d} x,
$$

see Proposition 2.1. On the other hand, the oscillations arising in the solutions to (HK), see Paragraph 3.1, show that (HK) can hardly be energy dissipating.

An obvious difference between (1.1) and (SH) is that the latter model does not take into account the erosion-deposition phenomena. Therefore, below, we compare the qualitative behaviour of the uppermost moving profile $u+h$ in (1.1) with its analog $h$ in (SH).

Then, we pass to the 1D case. After the standard preliminary study, we consider some numerical integrations of the different models and compare the corresponding solutions. It is immediate to see that as soon as a change in the slope of the bed is present, the two models (HK) and ( $\mathbf{S H})$ may display a somewhat surprising behavior. In particular, in the case of the former model, unphysical oscillations may arise in the short term and then disappear for large times, see Section 3.1. In the case of the latter model, when the slope changes sign, the sliding matter may accumulate creating unexpected peaks, see Section 3.2. This somewhat unphysical behaviour has to be expected, for the ( $\mathbf{S H})$ system is suited to bed whose slope has small variations.

The present model (1.1) and (HK) may differ also in the asymptotic behaviour, as shown in Section 3.3. There, the final profiles given by (1.1) and (HK) left from the fall of some granular material over a flat bed have in fact different concavities. 
The paper is organized as follows. First, in Section 2, we consider the main analytical properties of (1.1). Secondly, in Section 3, several numerical integrations show the main differences between the three models. The technical details are collected in Section 5 .

\section{Analytical Preliminaries}

This section is devoted to the analytical properties of the models. First, it is immediate to note that all systems are invariant with respect to the symmetry $x \rightarrow-x, t \rightarrow t, h \rightarrow h, v \rightarrow-v$ and $u \rightarrow u$, as it is physically necessary.

In the case of (1.3), we have the following energy dissipation property.

Proposition 2.1. Let $H=0$ and $V=0$. Choose smooth $\left(h_{o}, v_{o}, u_{o}\right)$ such that (1.3) with initial datum $\left(h_{o}, v_{o}, u_{o}\right)$ admits a smooth solution with compact support up to time $T>0$. Consider the energy (1.5). If (1.2) holds, then $E(h, v, u)(t)) \leq E\left(h_{o}, v_{o}, u_{o}\right)$ for all $t \in[0, T[$, more precisely,

$$
\begin{aligned}
\frac{d}{d t} E & =\int_{\mathbb{R}^{2}} h v \cdot \nu(v, p) \mathrm{d} x-\int_{\mathbb{R}^{2}} h\|v\|^{2}\left(|1-\|\nabla u\||-\frac{1}{2}(1-\|\nabla u\|)\right) \mathrm{d} x \\
& \leq 0 .
\end{aligned}
$$

The proof is immediate and, hence, omitted. Note that the energy decay has to terms: the former one is due to friction and the latter one to erosiondeposition.

Above, by smooth solution we mean that $(h, v, u) \in \mathbf{C}^{\mathbf{1}}\left(I \times \mathbb{R}^{2} ; \mathbb{R}^{+} \times\right.$ $\left.\mathbb{R}^{2} \times \mathbb{R}\right)$. However, as is well known, the smoothness of solutions does not persist, for singularities may arise. Therefore, we define a measurable map $(h, v, u): \mathbb{R}^{+} \times \mathbb{R}^{2} \mapsto \mathbb{R}^{+} \times \mathbb{R}^{2} \times \mathbb{R}$ to be a distributional solution to (1.3) if $(h, v, u)$ satisfies $(1.3)$ in the sense of distributions.

We now pass to the $1 \mathrm{D}$ case, so that (1.3) simplifies to

$$
\left\{\begin{array}{l}
\partial_{t} h+\partial_{x}(h v)=-\left(1-\left|\partial_{x} u\right|\right) h+H \\
\partial_{t} v+\partial_{x}\left(\frac{1}{2} v^{2}+h\right)=-\partial_{x} u+\nu\left(v, \partial_{x} u\right)-\llbracket 1-\left|\partial_{x} u\right| \rrbracket_{-} v+V \\
\partial_{t} u=\left(1-\left|\partial_{x} u\right|\right) h
\end{array}\right.
$$

and (1.4) to

$$
\left\{\begin{array}{l}
\partial_{t} h-\partial_{x}\left(h \partial_{x} u\right)=-\left(1-\left|\partial_{x} u\right|\right) h+H \\
\partial_{t} u=\left(1-\left|\partial_{x} u\right|\right) h
\end{array}\right.
$$


To study (2.1) and (2.2) as 1D systems of balance laws, it is useful to introduce the variable $p=\partial_{x} u$, obtaining

$$
\left\{\begin{array}{l}
\partial_{t} h+\partial_{x}(h v)=-(1-|p|) h+H \\
\partial_{t} v+\partial_{x}\left(\frac{1}{2} v^{2}+h\right)=-p+\nu(v, p)-\llbracket 1-|p| \rrbracket_{-} v+V \\
\partial_{t} p-\partial_{x}((1-|p|) h)=0
\end{array}\right.
$$

and, in the case of the Hadeler-Kuttler model,

$$
\left\{\begin{array}{l}
\partial_{t} h+\partial_{x}(h p)=-(1-|p|) h+H \\
\partial_{t} p-\partial_{x}((1-|p|) h)=0
\end{array}\right.
$$

Both these systems fall within the class of $3 \times 3$ systems of balance laws, see [7, Chapter VII] as a general reference on this subject.

In the case of distributional solutions, the equivalence between (2.1) and (2.3) is proved by the following lemma.

Lemma 2.2. Let $I=[0, T]$ for a $T>0$. If $(h, v, u)$ is a distributional solution to (2.1) satisfying

$$
\begin{gathered}
(h, v, u) \in \mathbf{L}^{\infty}\left(I \times \mathbb{R} ; \mathbb{R}^{+} \times \mathbb{R} \times \mathbb{R}\right), \\
\text { with } h(t) \text { and } \partial_{x} u(t) \in\left(\mathbf{L}^{1} \cap \mathbf{B V}\right)(\mathbb{R} ; \mathbb{R}) \quad \text { for a.e. } t \in I \\
\text { and } \partial_{x} u \in \mathbf{L}^{\infty}(I \times \mathbb{R} ; \mathbb{R})
\end{gathered}
$$

then, setting $p=\partial_{x} u$,

$$
\begin{gathered}
(h, v, p) \in \mathbf{L}^{\infty}\left(I \times \mathbb{R} ; \mathbb{R}^{+} \times \mathbb{R} \times \mathbb{R}\right), \\
\text { with } h(t) \text { and } p(t) \in\left(\mathbf{L}^{1} \cap \mathbf{B V}\right)(\mathbb{R} ; \mathbb{R}) \quad \text { for a.e. } t \in I
\end{gathered}
$$

is a distributional solution to (2.3). And viceversa.

The proof is deferred to Section 5. The equivalence of (1.4) and (2.4) is stated and proved similarly.

The first step in the analytical study of (2.3) is the computation of eigenvalues and eigenvectors of the Jacobian of the flow, which is the content of the next lemma.

Lemma 2.3. The Jacobian of the flow of system (2.3) is the matrix

$$
\left[\begin{array}{ccc}
v & h & 0 \\
1 & v & 0 \\
|p|-1 & 0 & h \operatorname{sgn} p
\end{array}\right]
$$


its eigenvalues and eigenvectors are

$$
\begin{gathered}
\lambda_{1}=v-\sqrt{h} \\
r_{1}=\left[\begin{array}{c}
\lambda_{2}=v+\sqrt{h} \\
-1 \\
+\frac{1}{\sqrt{h}} \\
\frac{1-|p|}{v-h \operatorname{sgn} p-\sqrt{h}}
\end{array}\right] \quad r_{2}=\left[\begin{array}{c}
\lambda_{3}=h \operatorname{sgn} p \\
\frac{1}{\sqrt{h}} \\
\frac{|p|-1}{v-h \operatorname{sgn} p+\sqrt{h}}
\end{array}\right] \quad r_{3}=\left[\begin{array}{l}
0 \\
0 \\
1
\end{array}\right] .
\end{gathered}
$$

If $p \neq 0, h>0$ and $v \neq h \pm \sqrt{h}$ then system (2.3) is strictly hyperbolic. Moreover,

$$
\nabla \lambda_{1} \cdot r_{1}=\frac{3}{2 \sqrt{h}}, \quad \nabla \lambda_{2} \cdot r_{2}=\frac{3}{2 \sqrt{h}}, \quad \nabla \lambda_{3} \cdot r_{3}=0 .
$$

so that the first two fields are genuinely nonlinear while the third one is linearly degenerate. For $p>0$, the ordering of the eigenvalues is as follows:

$$
\begin{aligned}
& \lambda_{3}<\lambda_{1}<\lambda_{2} \quad \Longleftrightarrow \quad v>h+\sqrt{h} \\
& \lambda_{1}<\lambda_{3}<\lambda_{2} \quad \Longleftrightarrow \quad h-\sqrt{h}<v<h+\sqrt{h} \\
& \lambda_{1}<\lambda_{2}<\lambda_{3} \quad \Longleftrightarrow \quad v<h-\sqrt{h}
\end{aligned}
$$

and symmetric relations hold for $p<0$.

The proof is straightforward and hence omitted. Remark that when $v=$ $h+\sqrt{h}$ then, not only $\lambda_{1}=\lambda_{3}$, but also the corresponding eigenspaces coincide, therefore hyperbolicity is lost. The same happens when $v=h-\sqrt{h}$. It is remarkable that, due to the loss of hyperbolicity at $h=0$ and to the form of the source term in (2.3), the well posedness of this system does not follow from the standard results on systems of balance laws. Indeed, fix any state $\left(h_{o}, v_{o}, u_{o}\right)$ where $(2.3)$ is strictly hyperbolic. Then, $\left(h_{o}, v_{o}, u_{o}\right)+\mathbf{L}^{1}(\mathbb{R} ; \mathbb{R})$ is not invariant with respect to the ordinary differential equation defined by the right hand side in (2.3). Nevertheless, given a positive $L$, there exists a $T>0$ such that the construction in [5] can be localized to any trapezoid of the type $\{(t, x) \in[0, T] \times \mathbb{R}:|x|<L+\hat{\lambda} t\}$. This procedure ensures the local well posedness of $(2.3)$.

For analytical results about $(2.4)$ we refer to $[1,18]$. Recall that it is a $2 \times 2$ system of balance laws with a Lipschitz flow, hyperbolic for $p \neq 0$. In $1 \mathrm{D}$, the Savage-Hutter model has the simpler form, see [15, formulæ (2.25)$(2.26)]$ :

$$
\left\{\begin{array}{l}
\partial_{t} h+\partial_{x}(h v)=0 \\
\partial_{t} v+\partial_{x}\left(\frac{1}{2} v^{2}+\delta \cos \zeta h\right)=\sin \zeta-\delta \cos \zeta \partial_{x} b+\nu \operatorname{sgn} v \cos \zeta
\end{array}\right.
$$



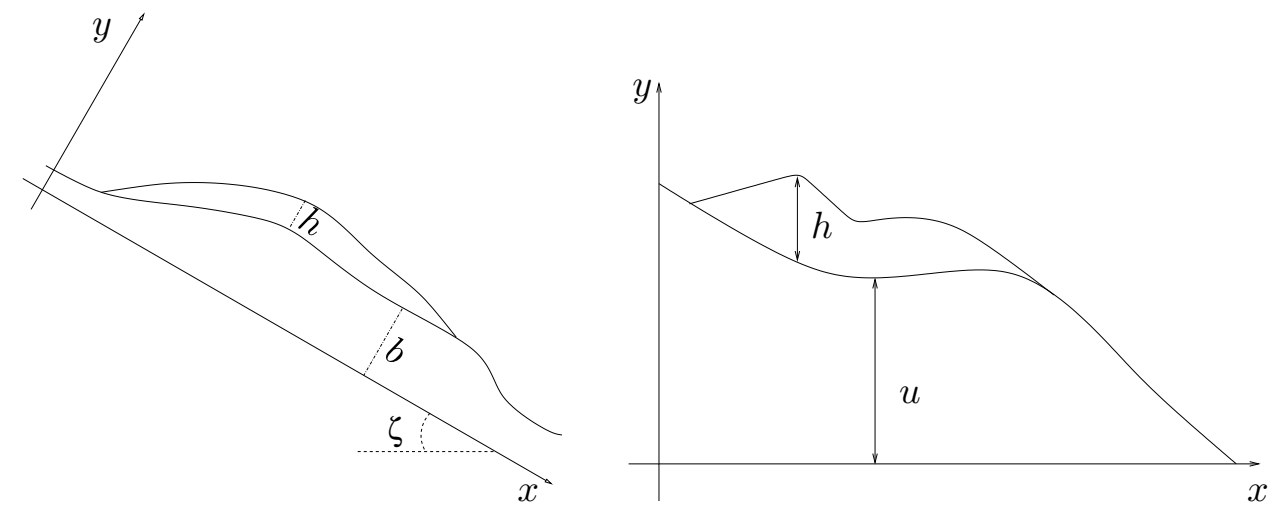

Figure 1: Left: notation for the 1D Savage-Hutter model (2.5). Right, notation for the 1D model (2.1).

where $\zeta$ is a constant slope angle, $\nu \operatorname{sgn}(v) \cos \zeta$ describes the friction of the sliding material with respect to the bed and $b$ describes the deviation of the bed from the constant angle $\zeta$, see Figure 2. In other words, the relation between the slope $u$ in (2.1) and the functions $b$ and $\zeta$ in (2.5) is

$$
u(x) \cos \zeta+x \sin \zeta=b(x \cos \zeta-u(x) \sin \zeta) .
$$

But, as already remarked, due to the absence of the erosion-deposition phenomena, in (2.5) $b$ and $\zeta$ are time independent, whereas $u$ is time dependent in $(2.1)$.

\section{Qualitative Behavior of the Solutions}

This section is devoted to various comparisons among the solutions to systems (2.3), with $\nu\left(v, \partial_{x} u\right)=-\nu v,(2.4)$ and (2.5). In all the numerical integrations, we use the standard Lax-Friedrichs method, see $[13, \S 12.5]$ coupled with Euler polygonals to deal with the source term, through the operator splitting method, see $[13, \S 17.1]$ or [5]. 


\subsection{Evolution of a Horizontal Profile}

As a first example, we consider the initial datum

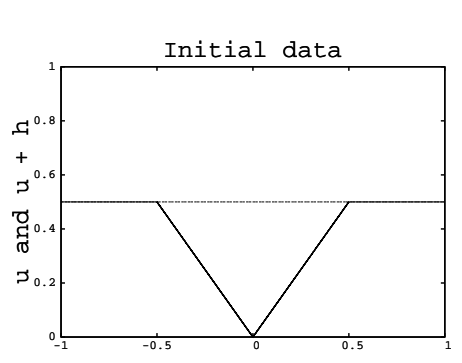

$$
\begin{aligned}
& h_{o}(x)= \begin{cases}0 & x>1 / 2 \\
\frac{1}{2}-|x| & x \in\left[-\frac{1}{2}, \frac{1}{2}\right] \\
0 & x<-1 / 2\end{cases} \\
& v_{o}(x)=0 \\
& p_{o}(x)= \begin{cases}0 & x<-1 / 2 \\
-1 & x \in[-1 / 2,0] \\
1 & x \in] 0,1 / 2] \\
0 & x>1 / 2\end{cases}
\end{aligned}
$$

which represents a hole filled with snow at rest. Choose $H=0$ and $V=0$.

Independently from the choice of $\nu$, the solution to (2.3)-(3.1) is stationary, which is physically reasonable. Indeed, where $h=0$ nothing may move. Where $h \neq 0$, the initial slope satisfies $|p|=1$ so that neither erosion nor deposition may take place. Besides, the effects of gravity disappear due to the fact that the profile is horizontal.

More formally, we prove the following lemma.

Lemma 3.1. Let $H=0, V=0$ and choose $\nu$ satisfying (1.2). Then, $(h, v, p)=\left(h_{o}, v_{o}, p_{o}\right)$ is a distributional stationary solution to (2.3)-(3.1).

The proof is in Section 5 .

On the contrary, the solution to (2.4)-(3.1) is not stationary and displays a somewhat unexpected behavior, depicted in Figure 2. Indeed, the change
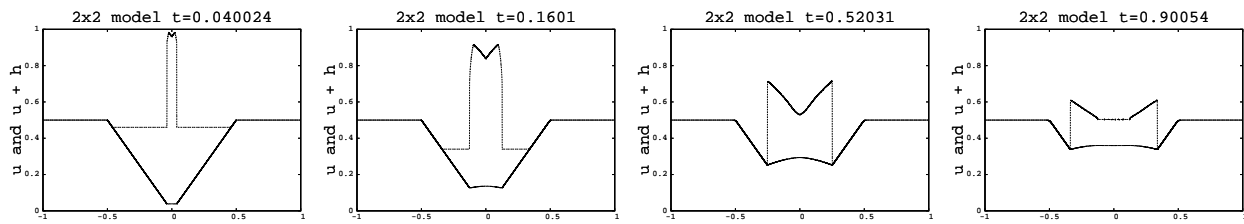

Figure 2: Integration of (2.4)-(3.1). The change in the slope at $x=0$ leads to the immediate formation of two large shocks which are eventually smeared out by the right hand side in (2.4).

in the slope at $x=0$ leads to the creation of two large shocks. These discontinuities are due to the convective part of (2.4), which dominates the source term at the small time scale. Consider the following Riemann problem 


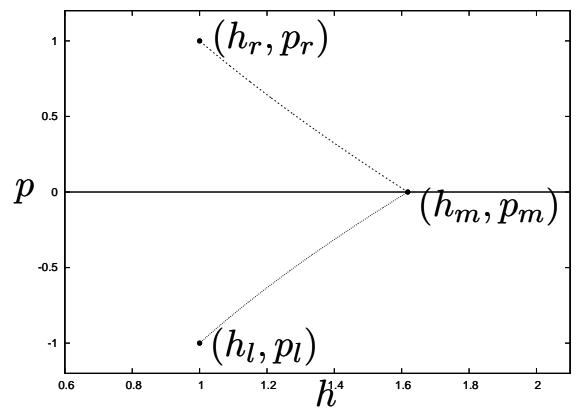

Figure 3: Shock curves, or Hugoniot loci, for to the solution of the Riemann problem (3.2), corresponding to the homogeneous part of (2.4) with the initial data (3.1). The solution to (3.2) governs that of (2.4)- (3.1) over the short time scale.

for the convective part of (2.4):

$$
\left\{\begin{array}{l}
\partial_{t} h+\partial_{x}(h p)=0 \\
\partial_{t} p-\partial_{x}((1-|p|) h)=0 \\
(h, p)(0, x)=\left\{\begin{array}{lll}
(1,-1) & x<0 \\
(1,1) & x>0
\end{array}\right.
\end{array}\right.
$$

Its solution consists of two (relatively) large shocks: see Figure 3 for the location of the Hugoniot loci displaying the solution to (3.2) and Figure 2, left, for the corresponding oscillations in the solution to (2.4)-(3.1). These shocks are eventually smeared out by the source terms and the solution to (2.4)-(3.1) approaches asymptotically the constant solution $h=0, p=0$.

We remark that the unphysical oscillations displayed in Figure 2 by the solutions to (2.4) are thus analytically justified consequences of the equations and are not due to numerical problems.

In this example, the asymptotic state reached by the solution to (2.3) differs from that of (2.4). However, in the case of the initial datum (3.1), this appears to be a non generic situation. Indeed, generically, small perturbations of the initial datum (3.1) lead to solutions of (2.3) that eventually tend to the asymptotic solution $h(x)=0, v=0, p=0$.

The rise of large shocks due to changes in the slope of the bed does not depend on the smoothness of this change. Indeed, consider the initial datum 


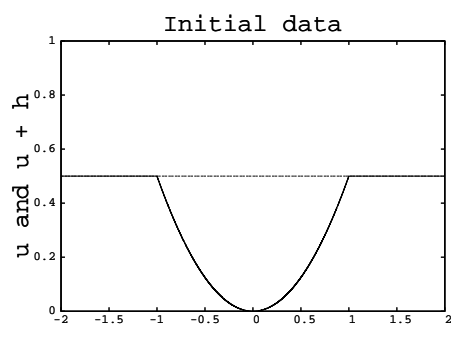

$$
\begin{aligned}
& h_{o}(x)= \begin{cases}0 & x>1 \\
\frac{1}{2}\left(1-x^{2}\right) & x \in[-1,1] \\
0 & x<-1\end{cases} \\
& v_{o}(x)=0 \\
& p_{o}(x)= \begin{cases}0 & x>1 \\
x & x \in[-1,1] \\
0 & x<-1\end{cases}
\end{aligned}
$$

representing a (smooth) hole filled with snow. We set $\nu=1, H=0$ and $V=0$. Then, the uppermost profile $u+h$ in the solution to (2.3)-(3.3) is again stationary, although deposition now takes place since $p$ attains values in ] $-1,1[: h$ diminishes to 0 and the sliding matter becomes part of the bed, see Figure 4.
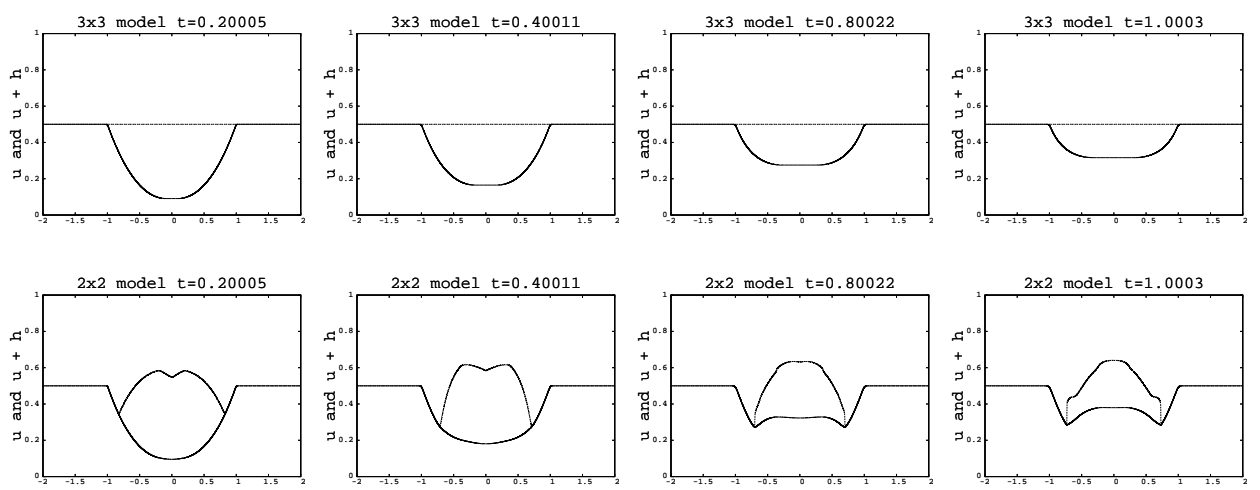

Figure 4: Above, the solution to (2.3)-(3.3): note that deposit takes place faster where the slope of the bed is lower. Below, the solution to (2.4)(3.3): note the formation of unexpected peaks near to $x=0$ where the slope smoothly changes sign.

On the other hand, in the case of (2.4), once more we have the shocks due to the convective part are present and lead to the formation of a sort of hill. The sliding matter accumulates at the center of the hole and its level gets higher than the initial one, see Figure 4.

Remark that asymptotically, the solutions to both models tend to $h=0$, $p=0$.

The solution to (2.5)-(3.3) is stationary and hence it is not displayed in Figure 4. Note that this behavior is physically acceptable, in spite of the fact that the Savage-Hutter model is adapted to describe only small variations in the average slope of the bed. 


\subsection{Falling Matter}

Consider now an avalanche or a landslide falling along a bed with varying slope. In other words we consider the initial datum:

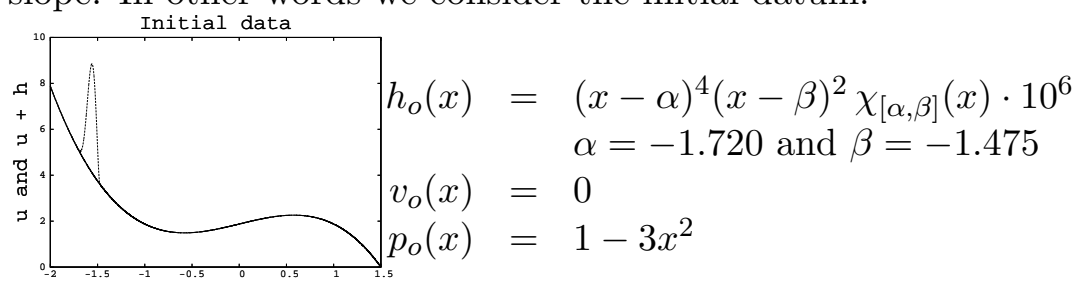

with $H=0, V=0$ and $\nu=0.1$. In (2.5) we also set $\delta=0.1, \zeta=0$ and $\partial_{x} b=p_{o}$.
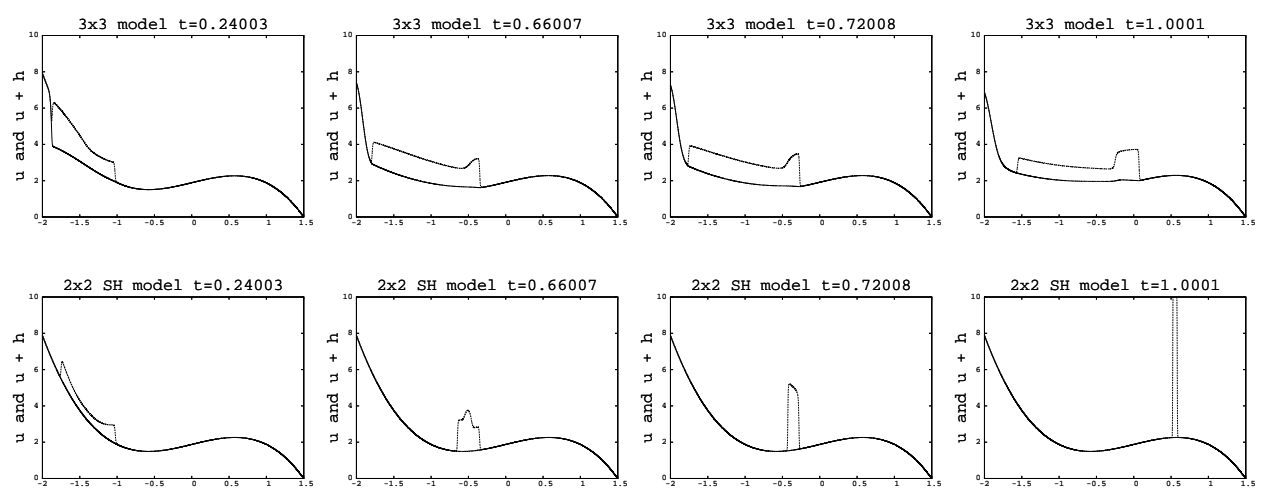

Figure 5: Above, integration of (2.3)-(3.4): first, the sliding matter erodes the bed while falling, then it deposits while slowing down. Below, integration of (2.5)-(3.4): neither erosion nor deposit may take place. Besides, the slower deceleration in the middle part causes the formation of a peak.

As long as the avalanche does not reach the change in the slope, the solution to (2.3) displays a bunch of matter moving downwards along the slope and, at the same time, eroding the steepest part of the bed. In the case of the Savage-Hutter model, the profile of the bed does not change, while that of the solution is quickly deeply modified, see Figure 5.

Where the bed's slope is small, the sliding matter in the solution to (2.3) slows down and starts depositing. In the solution to (2.5), by the absence of erosion-deposition term the sliding matter goes down faster than in the previous case, hence it concentrates and creates a peak, see Figure 5. 


\subsection{On the Role of $H$}

We now compare the three models (2.3), (2.4) and (2.5) in the case of a flat horizontal bed on which a material is being poured. Thus, assume that

$$
\begin{aligned}
& h_{o}(x)=0 \quad H(t, x)=1.5 \chi_{[-0.1,0.1]}(x) \cdot \chi_{[0,0.5]}(t) \\
& v_{o}(x)=0 \text { with } \quad V(t, x)=0 \\
& \begin{array}{ll}
p_{o}(x)=0 & \nu=0.1 .
\end{array}
\end{aligned}
$$

The results of the corresponding numerical integrations are collected in Figure 6 . We remark that, initially, the uppermost profiles $y=h(x)+u(x)$, in
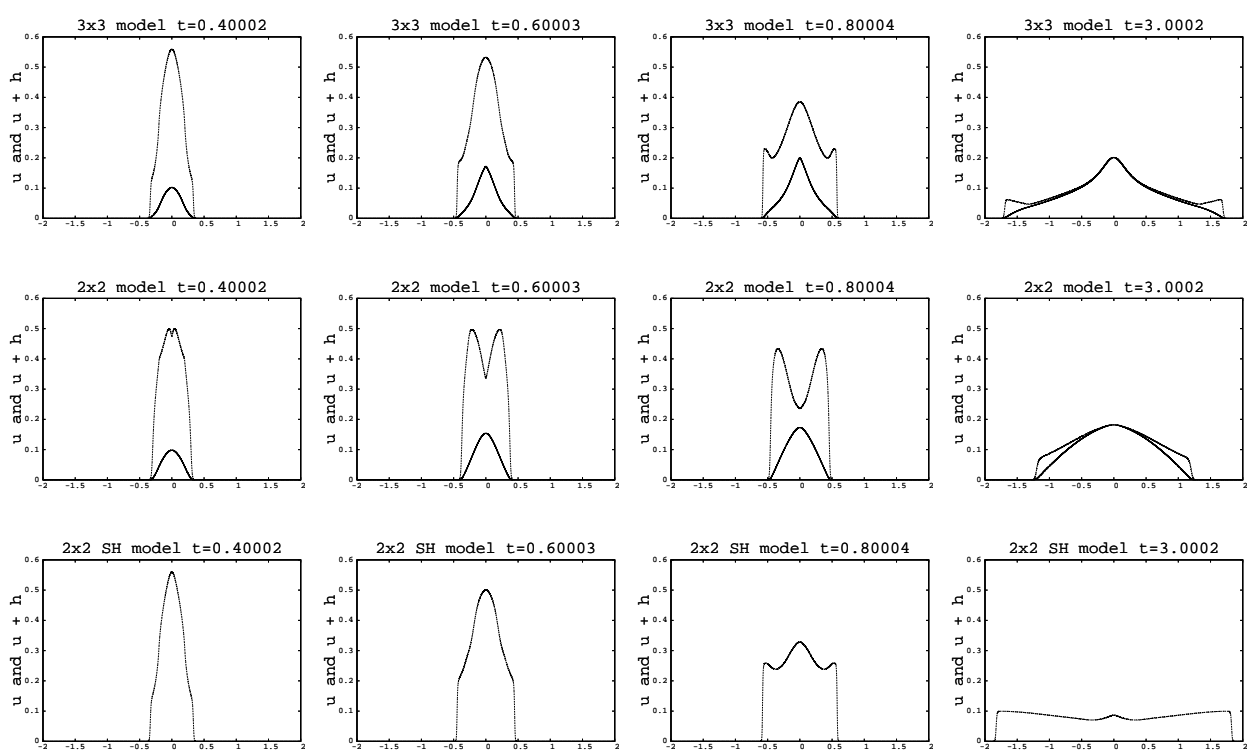

Figure 6: First row, solution to (2.3)-(3.5); second row, solution to (2.4)(3.5); third row, solution to (2.5)-(3.5). Note

the case of (2.3) and (2.4), $y=h(x)$, in the case of (2.5), are similar, see the first column in Figure 6. As soon as the lower deposited part takes a shape with a significant change in its slope, the solution to (2.4) displays the behaviour already noted above. Two symmetric shocks start moving off from the vertex of the deposited part, see the second and third columns in Figure 6.

Eventually, the solution to (2.5) spread all over the real line, for the (SH) model does not account for any deposit. However, as long as $h$ in (2.3) is positive, the qualitative aspects of the uppermost profiles in (2.3) and in (2.5) are analogous. Note that the asymptotic shape of the profile in the solutions to (2.3) and (2.4), rightmost column in Figure 6, are rather different. 


\section{Conclusions}

We presented a new model for the movement of granular matter. It is a synthesis of the Hadeler-Kuttler and of the Savage-Hutter models. The result is the $3 \times 3$ system (1.1), which we proved to be compliant with energy dissipation, similarly to the Savage-Hutter model, but able to describe the erosion-deposition dynamics, which is not considered in the (SH) model.

Moreover, (1.1) seems to describe better than the Hadeler-Kuttler model the evolution of the falling matter, in particular in case of changes in the bed slope. Indeed, the solutions to (1.1) do not display the sudden oscillations due to the convective part of (HK). Furthermore, we believe it is relevant that an initial horizontal profile evolves remaining horizontal, as in the cases examined in Paragraph 3.1.

\section{Technical Details}

We omit the proofs of the next two lemmas, since they are straightforward.

Lemma 5.1. [11, Appendix A] With the rescaling $x \rightarrow \frac{\beta}{\gamma} x, t \rightarrow \frac{1}{\alpha \gamma} t, u \rightarrow$ $\frac{\alpha \beta}{\gamma} u, h \rightarrow \frac{\alpha \beta}{\gamma} h$ and $f \rightarrow \frac{1}{\alpha^{2} \beta} f$, system (HK) reduces to (1.4).

Similarly, in the case of (1.1), we have the following lemma.

Lemma 5.2. With the rescaling $x \rightarrow \frac{g}{\alpha \gamma^{2}} x, t \rightarrow \frac{1}{\alpha \gamma} t, u \rightarrow \frac{g}{\gamma^{2}} u, h \rightarrow \frac{g}{\gamma^{2}} h$, $v \rightarrow \frac{g}{\gamma} v, H \rightarrow \frac{\gamma}{\alpha g} H, V \rightarrow \frac{\gamma^{2}}{\alpha g} V$ and $\nu \rightarrow \frac{\gamma^{2}}{\alpha g} \nu$ system (1.1) reduces to (1.3).

Proof of Lemma 2.2. Let $(h, v, u)$ be a distributional solution to (2.1) and set $p:=\partial_{x} u$. It is straightforward to show that the first two equations of (2.3) are satisfied in the sense of distributions. Moreover, for every test function $\varphi \in \mathbf{C}_{\mathbf{c}}^{\infty}\left(\mathbb{R}^{+} \times \mathbb{R} ; \mathbb{R}\right)$,

$$
\begin{aligned}
& \int_{\mathbb{R}^{+}} \int_{\mathbb{R}}\left[\partial_{t} \varphi p+\partial_{x} \varphi(1-|p|) h\right] \mathrm{d} x \mathrm{~d} t \\
= & \int_{\mathbb{R}^{+}} \int_{\mathbb{R}}\left[\partial_{t} \varphi \partial_{x} u+\partial_{x} \varphi\left(1-\left|\partial_{x} u\right|\right) h\right] \mathrm{d} x \mathrm{~d} t \\
= & \int_{\mathbb{R}^{+}} \int_{\mathbb{R}}\left[-\partial_{t} \partial_{x} \varphi u+\partial_{x} \varphi\left(1-\left|\partial_{x} u\right|\right) h\right] \mathrm{d} x \mathrm{~d} t .
\end{aligned}
$$

Now, using the fact that $\partial_{x} \varphi$ belongs to $\mathbf{C}_{\mathbf{c}}^{\infty}\left(\mathbb{R}^{+} \times \mathbb{R} ; \mathbb{R}\right)$ and the hypothesis that $(h, v, u)$ satisfies the latter equation of $(2.1)$, we get

$$
\int_{\mathbb{R}^{+}} \int_{\mathbb{R}}\left[\partial_{t} \varphi p+\partial_{x} \varphi(1-|p|) h\right] \mathrm{d} x \mathrm{~d} t=0,
$$

i.e. also the third equation of (2.3) holds in the distributional sense. 
Let $(h, v, p)$ be a distributional solution to $(2.3)$ and define $u(t, x):=$ $\int_{-\infty}^{x} p(t, \xi) \mathrm{d} \xi$. As above, the proof for the first two equations is trivial. Moreover, using (2.3) and [7, Theorem 4.3.1]:

$$
\begin{aligned}
& \int_{a}^{x} p(t, \xi) \mathrm{d} \xi-\int_{a}^{x} p(0, \xi) \mathrm{d} \xi \\
= & \int_{0}^{t}(1-|p(\vartheta, x-)|) h(\vartheta, x-) \mathrm{d} \vartheta-\int_{0}^{t}(1-|p(\vartheta, a+)|) h(\vartheta, a+) \mathrm{d} \vartheta .
\end{aligned}
$$

Since $h(t)$ and $p(t)$ belong to $\left(\mathbf{L}^{1} \cap \mathbf{B V}\right)(\mathbb{R} ; \mathbb{R})$ for a.e. $t \in I$, when $a \rightarrow-\infty$ we get:

$$
u(t, x)=\int_{-\infty}^{x} p(t, \xi) \mathrm{d} \xi=\int_{-\infty}^{x} p(0, \xi) \mathrm{d} \xi+\int_{0}^{t}(1-|p(\vartheta, x)|) h(\vartheta, x) \mathrm{d} \vartheta .
$$

Introduce, for simplicity, the quantities $g(x):=\int_{-\infty}^{x} p(0, \xi) \mathrm{d} \xi$ and $l(t, x):=$ $\int_{0}^{t}(1-|p(\vartheta, x)|) h(\vartheta, x) \mathrm{d} \vartheta$. Then, for every test function $\varphi \in \mathbf{C}_{\mathbf{c}}^{\infty}\left(\mathbb{R}^{+} \times\right.$ $\mathbb{R} ; \mathbb{R})$,

$$
\begin{aligned}
& \int_{\mathbb{R}^{+}} \int_{\mathbb{R}}\left[\partial_{t} \varphi u+\varphi\left(1-\left|\partial_{x} u\right|\right) h\right] \mathrm{d} x \mathrm{~d} t \\
= & \int_{\mathbb{R}^{+}} \int_{\mathbb{R}}\left[\partial_{t} \varphi g+\partial_{t} \varphi l+\varphi(1-|p|) h\right] \mathrm{d} x \mathrm{~d} t .
\end{aligned}
$$

Now, integrating by parts and using the fact that $\partial_{t} g=0$ and $\partial_{t} l=$ $(1-|p|) h$, we obtain:

$$
\int_{\mathbb{R}^{+}} \int_{\mathbb{R}}\left[\partial_{t} \varphi u+\varphi\left(1-\left|\partial_{x} u\right|\right) h\right] \mathrm{d} x \mathrm{~d} t=0 .
$$

Hence, also the third equation in (2.1) is satisfied in distributional sense and the proof is completed.

Proof of Lemma 3.1. Let $h, v, p$ be the stationary functions $h(t, x)=$ $h_{o}(x), v(t, x)=0, p(t, x)=p_{o}(x)$. Separately, in each of the regions $\mathbb{R}^{+} \times$ ]$-\infty,-1 / 2\left[, \mathbb{R}^{+} \times\right]-1 / 2,0\left[, \mathbb{R}^{+} \times\right] 0,1 / 2\left[\right.$ and $\left.\mathbb{R}^{+} \times\right] 1 / 2,+\infty[,(h, v, p)$ is a smooth solution to $(2.3)$. On the other hand, the traces of $(h, v, p)$ along the three boundaries $\mathbb{R}^{+} \times\{-1 / 2\}, \mathbb{R}^{+} \times\{0\}$ and $\mathbb{R}^{+} \times\{1 / 2\}$, satisfy the Rankine-Hugoniot conditions with zero speed. Hence $(h, v, p)$ is a stationary solution.

\section{References}

[1] D. Amadori and W. Shen. Global existence of large BV solutions in a model of granular flow. Preprint 2008, 63(1):259-278 (electronic), 2002. 
[2] P. Cannarsa and P. Cardaliaguet. Representation of equilibrium solutions to the table problem for growing sandpiles. J. Eur. Math. Soc. (JEMS), 6(4):435464, 2004.

[3] P. Cannarsa, P. Cardaliaguet, G. Crasta, and E. Giorgieri. A boundary value problem for a PDE model in mass transfer theory: representation of solutions and applications. Calc. Var. Partial Differential Equations, 24(4):431-457, 2005.

[4] P. Cannarsa, P. Cardaliaguet, and E. Giorgieri. Hölder regularity of the normal distance with an application to a PDE model for growing sandpiles. Trans. Amer. Math. Soc., 359(6):2741-2775 (electronic), 2007.

[5] R. M. Colombo and G. Guerra. Hyperbolic balance laws with a non local source. Comm. Partial Differential Equations, 32(10-12):1917-1939, 2007.

[6] G. Crasta and S. Finzi Vita. An existence result for the sandpile problem on flat tables with walls. Netw. Heterog. Media, 3(4):815-830, 2008.

[7] C. M. Dafermos. Hyperbolic conservation laws in continuum physics, volume 325 of Grundlehren der Mathematischen Wissenschaften [Fundamental Principles of Mathematical Sciences]. Springer-Verlag, Berlin, second edition, 2005.

[8] A. de Saint-Venant. Thorie du mouvement non-permanent des eaux, avec application aux crues des rivires et l'introduction des mares dans leur lit. C.R.Acad.Sci.Paris 73, 1871.

[9] M. Falcone and S. Finzi Vita. A finite-difference approximation of a twolayer system for growing sandpiles. SIAM J. Sci. Comput., 28(3):1120-1132 (electronic), 2006.

[10] P. Gwiazda and A. Świerczewska. Multivalued equations for granular avalanches. Nonlinear Anal., 62(5):895-912, 2005.

[11] K. Hadeler and C. Kuttler. Dynamical models for granular matter. Granular Matter, 2(9-18), 1999.

[12] K. Hadeler and C. Kuttler. Granular matter in a silo. Granular Matter, 3(193197), 2001.

[13] R. J. LeVeque. Finite volume methods for hyperbolic problems. Cambridge Texts in Applied Mathematics. Cambridge University Press, Cambridge, 2002.

[14] M. Pelanti, F. Bouchut, and A. Mangeney. A Roe-type scheme for two-phase shallow granular flows over variable topography. M2AN Math. Model. Numer. Anal., 42(5):851-885, 2008.

[15] S. B. Savage and K. Hutter. The motion of a finite mass of granular material down a rough incline. J. Fluid Mech., 199:177-215, 1989.

[16] S. B. Savage and K. Hutter. The dynamics of avalanches of granular materials from initiation to runout. I. Analysis. Acta Mech., 86(1-4):201-223, 1991.

[17] M. Shearer, J. M. N. T. Gray, and A. R. Thornton. Stable solutions of a scalar conservation law for particle-size segregation in dense granular avalanches. European J. Appl. Math., 19(1):61-86, 2008. 
[18] W. Shen. On the shape of avalanches. J. Math. Anal. Appl., 339(2):828-838, 2008.

[19] Y. Wang, K. Hutter, and S. P. Pudasaini. The Savage-Hutter theory: a system of partial differential equations for avalanche flows of snow, debris, and mud. ZAMM Z. Angew. Math. Mech., 84(8):507-527, 2004. 